\title{
The Digital Twin as a Mediator \\ for the Digitalization and Conservation \\ of Expert Knowledge
}

\author{
Dominik Hüsener, Michael Schluse, Dorit Kaufmann \\ and Jürgen Roßmann
}

\begin{abstract}
A Digital Twin is a virtual representation of a physical asset. It reflects the current state of that machine through a model and the data as observed by sensors in the real machine; and enables effective and efficient interaction with the machine, i.e. for monitoring and control purposes. The Digital Twin facilitates the collection of data, as well as its analysis and visualization through its user interfaces, i.e. GUIs such as screens or Mixed Reality that provide intuitive access to the data and facilitates its manipulation. Embedded in Virtual Testbeds the Digital Twin becomes an "Experimentable Digital Twin" (EDT), in which experiments can be performed and the different outcomes can be compared or evaluated. The intuitive representation of the assets allows the experts to interact with the twin, without highly detailed knowledge in computer science. The digital twin observes, records, and benchmarks experiments performed by the operator. This way the operator's knowledge becomes digitized and thus preserved as an abstract representation of data, formulas, and models inside the digital twin. By introducing the Digital Twin into the processes carried out by different operators (not only the initially observed expert), formerly intuitive decision-making processes of the operators are enhanced based on empirical data. As a result, the Digital Twin serves as an assistance system that can guide future operators and the outcomes
\end{abstract}

D. Hüsener $(\bowtie) \cdot$ M. Schluse $\cdot$ D. Kaufmann · J. Roßmann Institute for Man-Machine Interaction, RWTH Aachen University, Ahornstr. 55, 52074 Aachen, Germany

e-mail: huesener@mmi.rwth-aachen.de

T. Schüppstuhl et al. (eds.), Annals of Scientific Society for Assembly,

Handling and Industrial Robotics 2021,

https://doi.org/10.1007/978-3-030-74032-0_20 
of the experiments become reproducible. The specific representations of interactions and outcomes also facilitate collaboration between the machine operators and other stakeholders by providing different operators a common "perspective".

\section{Keywords}

Digital twin $\cdot$ Decision making $\cdot$ Knowledge digitalization

\section{Introduction}

With the advance of Industry 4.0, there is a shift from mass production to innovative, specialized products with small batch sizes. Therefore, machine parameters need to be changed more frequently. In a traditional production setting, this leads to higher personnel, time, and material requirements and consequently higher production costs [1]. In some cases, the new settings need to be tested before the actual production can begin further increasing time and cost requirements. Even if the test pieces are within tolerances, the final product can be faulty. On older machines, which are still in use in some industry sectors, machine parameters need to be adjusted manually for each product, thus limiting the possibility of batch size 1 and driving costs up for small batch sizes.

Especially in some disciplines belonging to the traditional mechanical engineering sector (as textile mechanical engineering), the success of the outcome is depending on the skills of the operator and his experience in setting special machine parameters. Thus, effective production is only possible with experienced, skilled personnel. This knowledge is not measurable and thus not transformable. This problem is enhanced by a lack of young employees, trainees, and skilled workers, and the changes in demographics [10].

The knowledge however is not conserved but is in the heads of the employees and depends on word of mouth to be passed along. This is because knowledge is empirical and not quantized. When an employee leaves the company, the knowledge is gone. Due to the limited use of digitalization in some industry sectors, the training takes long, and even skilled workers need time to learn to use an individual machine independently.

The rest of the paper is organized as follows. In Sect. 2 the Digital Twin concept is presented with the integration of simulation in a so-called Experimentable Digital Twin and mentioning its role as a mediator. In Sect. 3, a brief overview of knowledge management is given and combined with the Digital Twin approach as a means of knowledge management. In Sect. 4, this concept is transferred to a use case of the textile industry. 


\section{What is a Digital Twin?}

The Digital Twin (DT) was first presented by Grieves [3] as a virtual model of a physical asset that receives data from the asset and virtually represents the state of the asset. In 2003 the "Digital Twin" concept was first presented. In 2014 Grieves published a white paper on the Digital Twin. A Digital Twin is a virtual representation of an asset that is fed with data from the real machine and also sends data to the real twin. The DT can be obtained by combining the data of the machine (the Digital Shadow) with a model of the machine (see Fig. 1).

The combination of the physical asset and the Digital Twin with its corresponding communication infrastructure is called a Cyber-Physical System (CPS). The physical asset can itself be a CPS. In Industry 4.0 CPS become increasingly important. Different machines have their own twin, while the twins can communicate with each other. The Digital Twin is used in health monitoring, also production planning (PLM), and the design of new products. In comparison to conventional planning software, what is new is that all data related to one product is stored together in one place (the Digital Twin) whereas before machines were able to store data but it was not combined into one unified presentation. This presentation however has brought a massive increase in value. Although it is only a simplified representation, it tries to model the behavior of the real twin accurately.

In Kritzinger et al. [5] literature on the Digital Twin in the manufacturing field is reviewed and classified as either Digital Model, Digital Shadow, or Digital Twin.

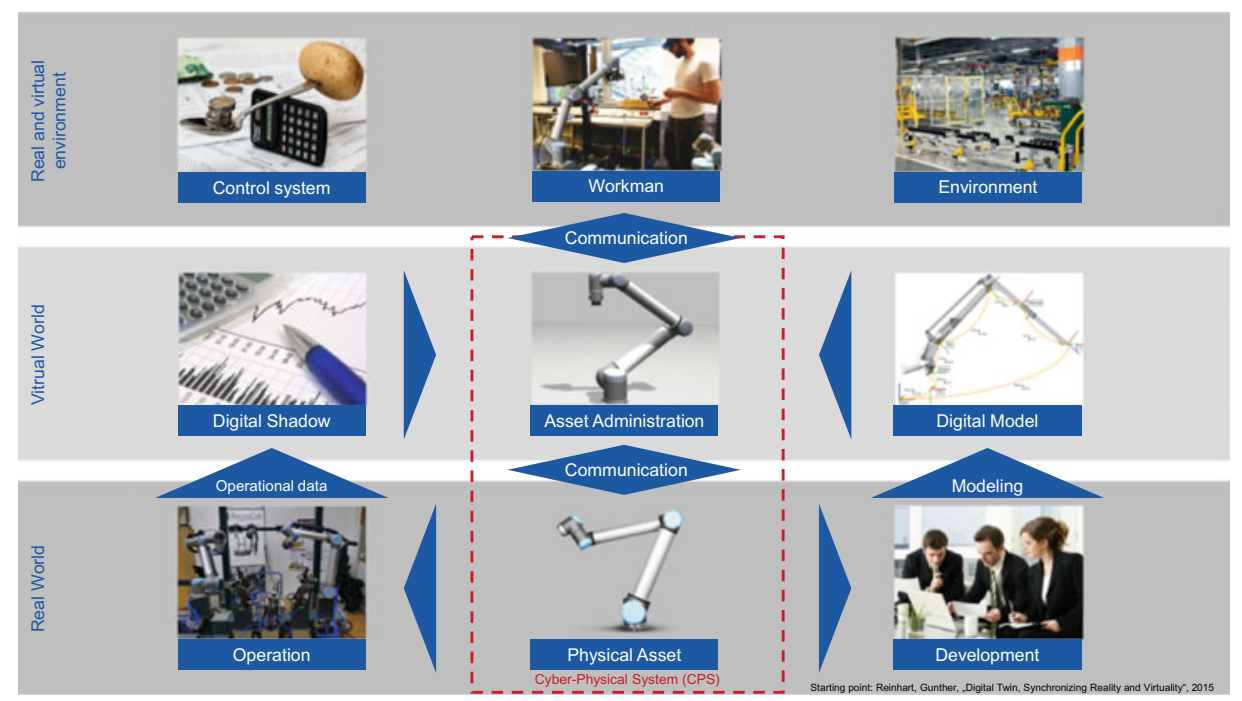

Fig. 1 The context of the Experimentable Digital Twin (EDT) and the Cyber-Physical System (CPS) [7, 8] 
According to their definition, the difference with these terms is the communication between the digital and physical assets. Whereas the Digital Model is independent of the real asset, the Digital Shadow only receives data from the real asset and only the Digital Twin allows bi-directional communication between digital and physical assets. While the term "Digital Twin" is widely used in literature, it is often used for Digital Models and Shadows as well. Therefore, the need for a common definition of the Digital Twin is emphasized. The literature on actual Digital Twins according to their proposed definition however is scarce. Especially there is a lack of case studies on a higher level of integration.

A more recent literature review was performed in Tao et al. [11] with a focus on applications of the Digital Twin in industry. After the white paper of Grieves, the number of papers published is increasing steadily. It is also mentioned, that the definition of the Digital Twin is varying in different papers. The most relevant theoretical foundation of the DT is listed as DT modeling, simulation, verification, validation, and accreditation (VV\&A); data fusion; interaction and collaboration; and service. The main applications currently where the DT is used are production and health management.

\subsection{What is an Experimentable Digital Twin?}

The experimentable Digital Twin brings together modern simulation technologies with the Digital Twin concept [9]. Whereas before several simulations (FEM, fluid dynamics, multi-body dynamics...) were performed individually, this can lead to wrong results if there is no exchange between the results. The Digital Twin enabled by co-simulation brings together different simulation techniques and stores the relevant data in the Digital Twin such that is exchanged between the various simulations (see Fig. 2).

With simulation, it is not only possible that a Digital Twin supervises a system and has a virtual representation but also a view into the future becomes feasible. The Digital Twin is not only able to collect the machine data but also to generate new data and through this improve the functionality of the physical asset. In manual production processes, it is difficult to use artificial intelligence to optimize the process since data of the machine and the produced object are not stored together. The Digital Twin however also opens the door to artificial intelligence techniques that can optimize the behavior.

\subsection{Digital Twin as a Mediator}

The Digital Twin displays its current status and can suggest actions to be taken through the human-machine interface. The machine sends its data which is processed by the Digital Twin to display valuable information to the user in an understandable way. The operator sees the data, can understand its meaning and can act accordingly (see Fig. 3). 


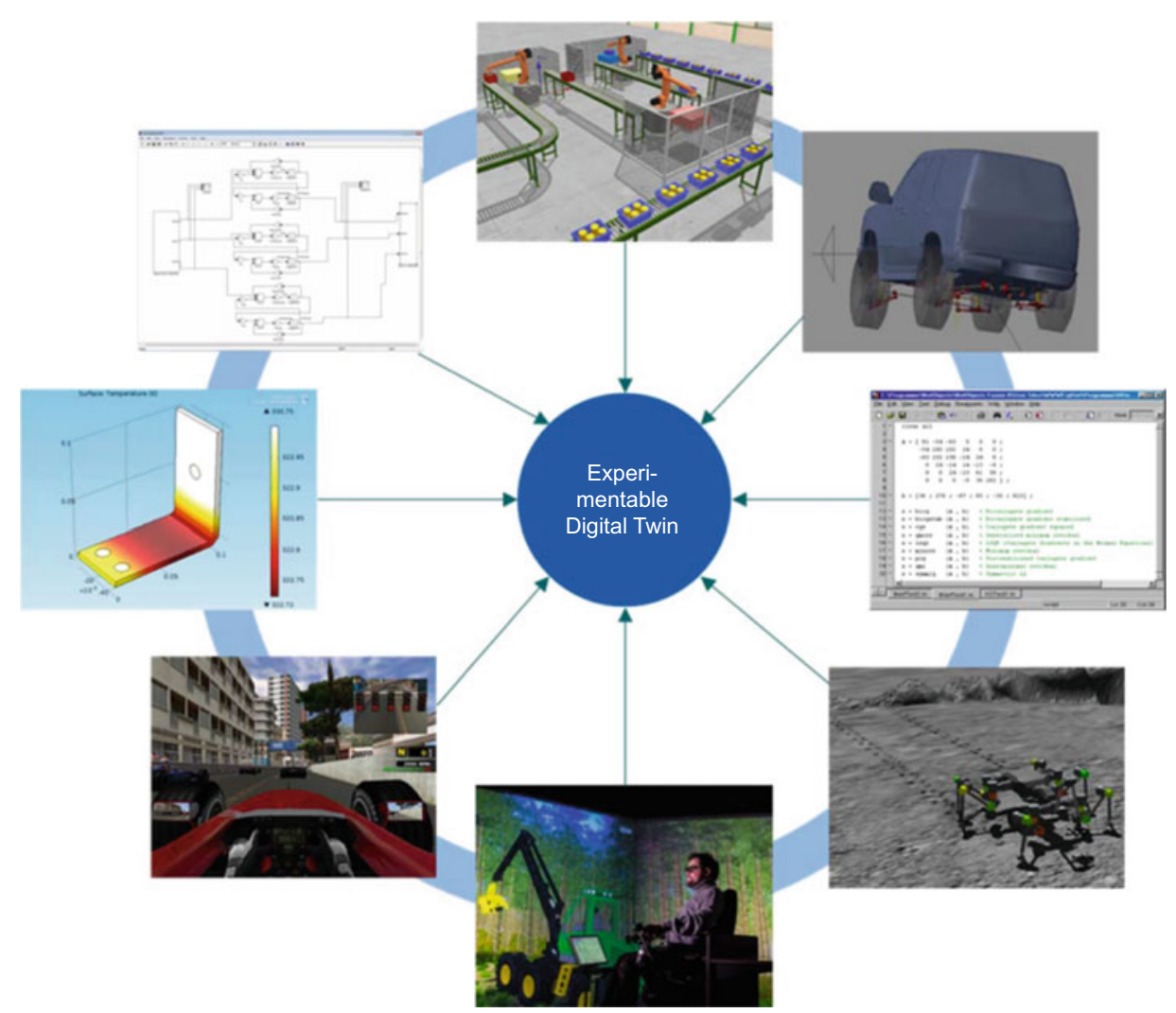

Fig. 2 "Experimentable Digital Twin approach". The EDT combines several simulation algorithms [9]

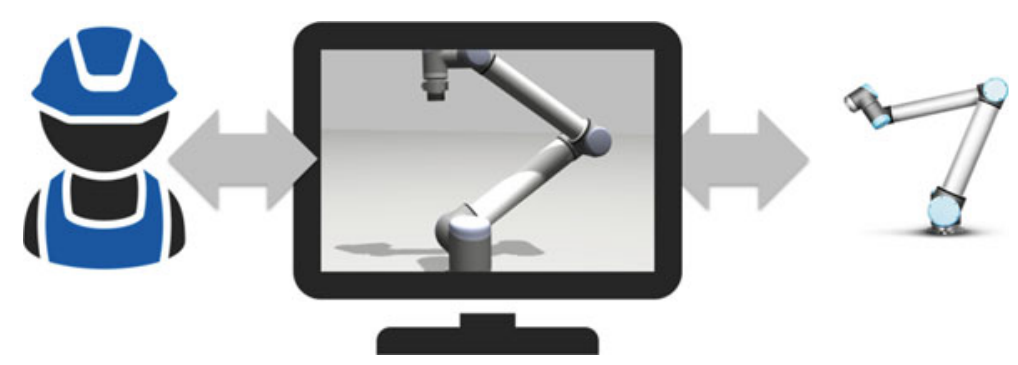

Fig. 3 Schematic concept of the digital twin as a mediator between the operator and the real twin

In his whitepaper Grieves [3] describes, how the virtual representation is beneficial to conceptualize, compare and collaborate in production processes since they provide a more intuitive perspective compared to $2 \mathrm{D}$ sketches or data in tables or regular graphs. The realistic visual view is valuable since sight is the most important human sense. Different 
users can see a representation that easily allows them to classify the current state of the machine which is also consistent for different users [4].

The user can simulate the behavior of the machine and is supported to find suitable settings for a given task. The simulation can be displayed in 3D and information that is otherwise not visible to the operator can be visualized through the Digital Twin. Hence, the Digital Twin can also be regarded as a mediator between the machine operator and the machine itself. In Cichon and Rossmann [2] a concept to facilitate the interaction of humans and machines is presented. Among others, joysticks, screens and AR/VR allow direct interaction with the digital twin. The user is able to interact with the virtual machine similar to the real machine, and the (real) machine informs the user of its current status through the visualization of the Digital Twin.

\section{What is Knowledge Management?}

Knowledge management is about conserving the (procedural) knowledge that is needed to perform a certain task. As mentioned earlier, the aging workforce requires that their knowledge is somehow stored in order not to lose the knowledge which is an essential asset for the company to remain productive. This is traditionally performed manually through observational studies and questionnaires [6]. Questionnaires however require the person conducting the study to have some basic knowledge of the process to be considered in order to ask the relevant questions. Otherwise, some necessary or useful information might be left out. Also, questionnaires require additional work to provide a benefit. They are only useful if the results are presented in a manner such that they are comprehensible to trainees. A textual description for example can be useful for experienced workers that need to learn how to operate a new machine but might be difficult to grasp for students that are not yet familiar with the functioning of the machine.

Observational studies are better in this way; however, it becomes costly very quickly if there is a high amount of different work tasks to do and infeasible to record tasks that only occur seldomly. Also, this requires a setup and is not simply integrated into the regular working process.

In Mohammad and Al Saiyd [6] a process for manually acquiring expert data in the context of artificial intelligence is presented. The required activities are the identification of the domain knowledge, finding experts that can provide that knowledge, finding a representation for modeling of that knowledge, and the construction of a knowledge base that can then be used in an inference engine (see Fig. 4). 
Fig. 4 Activities of knowledge engineering and the integration of the EDT [6]

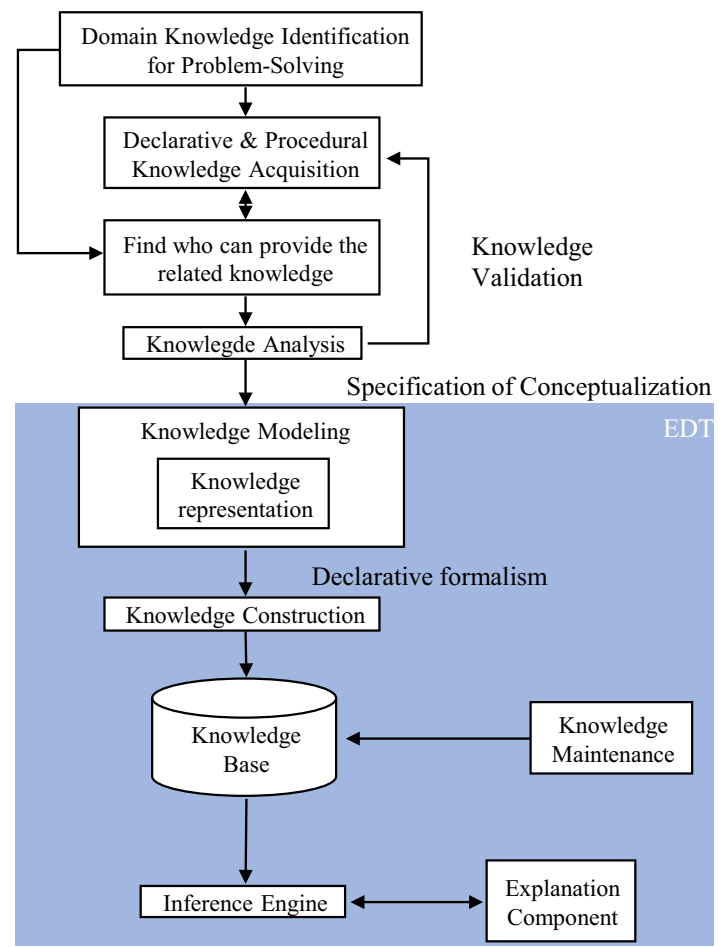

\subsection{How to Conserve Knowledge with a Digital Twin}

If the knowledge of the workforce is to be conserved, it can be digitized in some way. Digitization has many advantages and can increase the value of the captured information when it is combined with other information. Considering the activities in Mohammad and Al Saiyd [6], we propose to use the Digital Twin as a means of knowledge representation and to store the knowledge base. The Digital Twin also receives real-time data from the machine - as a result, there is one central data storage for all information related to that machine ("Single Source of Truth"). The Digital Twin can store data like a conventional database, but it also has the advantage of being able to visualize that data more intuitively. A further advantage of the Digital Twin is that at the end of the process a virtual model is generated that can be verified by the experts. Also, the virtual representation can help trainees as processes can be shown on a 3D model that - other than a video — can also be manipulated and is easier to grasp than a textual description. Through the use of augmented reality, it could even be possible to see a virtual representation of the machine along with the real machine that can guide the worker through the process of adjusting the machine parameters or maintenance with the advantage of having their hands free. 
However, the data of the interaction is stored by the machine and transferred into the database of the Digital Twin, it becomes feasible to record processes on the go and understandably conserve them. Also, not very frequent tasks are conserved this way. The digital twin itself is subject to an ageing process, hence it needs to adapt to new production environments in order to sustain its relevance.

\section{Use Case}

The project 'Development of an experimentable digital twin for the analysis and automated adaptation of textile manufacturing processes using the example of tufting technology (T-EXDIZ)' aims to show in the example setting of a textile machine how the Digital Twin can bring benefit to manual tasks that are difficult to automize. The machines are usually kept for decades before they are replaced, and older machines cannot be automized easily. It is however possible to include sensors into the machine, such that various parameters (e.g. angle, length) of machine parts or settings can be read. To improve automation, the Digital Twin can record these settings and thus create a virtual representation of the process. The operator can experiment with the Digital Twin, allowing him to experience the consequences of his activities. These steps can be visualized on a screen through a virtual representation of the Digital Twin (i.e. rendering) which is usually a simplified representation of the real twin. Hence, through utilization, the settings are kept for further use. It is then possible to test these new settings using the simulation methodology without having to waste material. If this product has to be produced again the exact values are stored and can be compared to the current values such that reproducibility is conserved. Besides, this also makes it possible to teach the process to new trainees. The Digital Twin can also simulate its behavior to provide training opportunities. Also, there is an opportunity to test new configurations without the costly procedure of testing each setting since the simulation can perform this testing in a fracture of the time. It can thus help trainees gain confidence and reduce the amount of training needed to operate the machine.

If a worker leaves the company, his procedural knowledge can be stored in an understandable form such that it is available to new trainees. Also, for experienced workers, it becomes simpler to check whether they have completed steps correctly. The human-machine interface (HMI) - a window in the virtual world where the digital twin lives - can help to visualize what steps must be taken. The spread by word-and-mouth also relies on a common understanding, the Digital Twin however has one unified representation for everyone. In training, it will be more easily possible to test multiple configurations.

This procedure is shown in the use case of a textile machine. In the textile industry automation is low and batch size one production is often not feasible. The computer has a model of the machine that can be used to simulate the machine. It receives data from the operation of the machine. The real data can be used to update simulation parameters and it 
Fig. 5 The DT concept applied to the T-EXDIZ use case

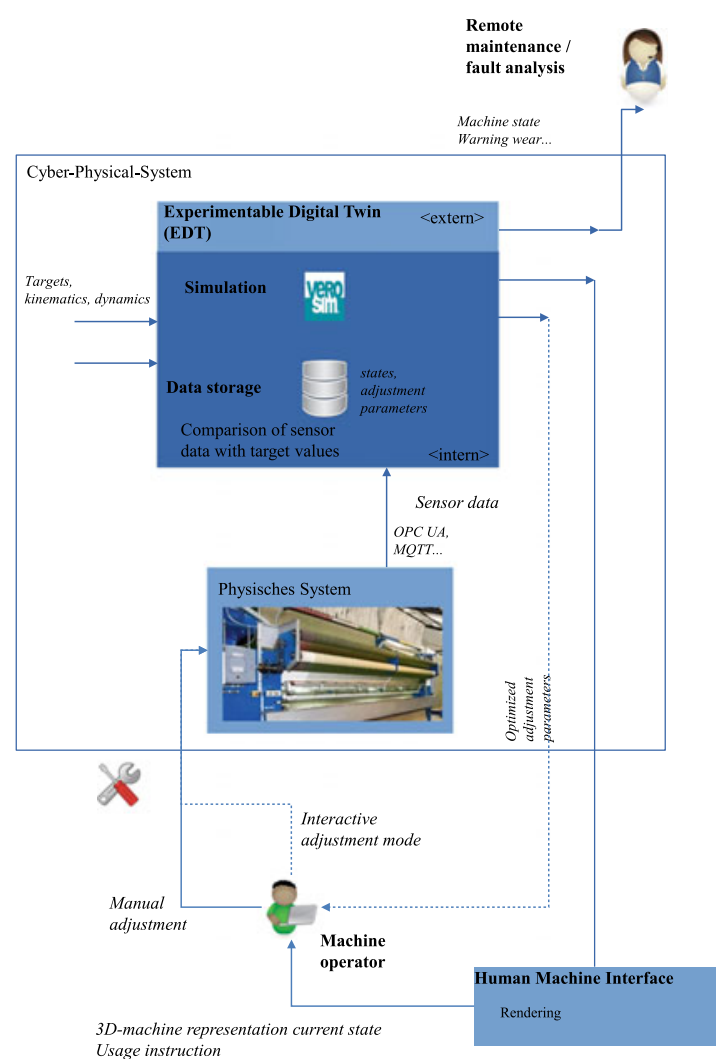

can also be used to detect irregularities and advise the worker to repair machine parts or to adjust settings. Instead of waiting for the finished product and noticing that it does not fulfill its specifications, the Digital Twin can simulate further behavior based on the current state. Thus, faults can be detected early and waste products, and thus cost is decreased.

The flow of data and information between the different components is shown in Fig. 5.

Currently, a DT model of the kinematics of a tufting machine is created. In the next step, this model can be refined with the input of experts. Later an automated mode will be integrated that can optimize machine parameters through simulation transforming the DT to an experimentable Digital Twin.

\section{Conclusion}

Due to an aging workforce, some personnel is leaving the production sites in the coming years. However, the knowledge of the workers is an important asset for the company to remain competitive, especially in sectors where manual labor is still widely present. 
The Digital Twin is a virtual model of an asset that can be used to store and visualize machine data. As was shown in this paper, the Digital Twin can also be used to conserve the knowledge of the workforce and make it measurable in the first place. This helps in reducing training times for new employees and knowledge loss. The knowledge that comes from questionnaires, as well as observations, cannot only increase the value of the Digital Twin itself but the DT is also a useful tool to have a meaningful representation of that knowledge.

Acknowledgements The writing of this chapter was enabled within the context of the IGF project 'Development of an experimentable digital twin for the analysis and automated adaptation of textile manufacturing processes using the example of tufting technology (T-EXDIZ)' $21166 \mathrm{~N} / 2$ of the Forschungsvereinigung Forschungskuratorium Textil e.V.. It is funded via the AiF within the framework of the program for the promotion of joint industrial research and development (IGF) by the Federal Ministry of Economics and Energy based on a resolution of the German Bundestag.

\section{References}

1. Allahverdi, A., Soroush, H.M.: The significance of reducing setup times/setup costs. Eur. J. Oper. Res. 187, 978-984 (2008). https://doi.org/10.1016/j.ejor.2006.09.010

2. Cichon, T., Rossmann, J.: Digital twins: assisting and supporting cooperation in human-robot teams. In: Proceedings of the 15 th International Conference on Control, Automation, Robotics and Vision (ICARCV 2018), November 18-21, 2018, Singapore, pp. 1-6 (2019)

3. Grieves, M.: Digital twin: manufacturing excellence through virtual factory replication. White Pap. 1, 1-7 (2014)

4. Grieves, M., Vickers, J.: Digital twin: mitigating unpredictable, undesirable emergent behavior in complex systems. In: Transdisciplinary Perspectives on Complex Systems, pp. 85-113. Springer, Cham (2017). https://doi.org/10.1007/978-3-319-38756-7_4

5. Kritzinger, W., Karner, M., Traar, G., Henjes, J., Sihn, W.: Digital twin in manufacturing: a categorical literature review and classification. IFAC-PapersOnLine. 51, 1016-1022 (2018). https://doi.org/10.1016/j.ifacol.2018.08.474

6. Mohammad, A., Al Saiyd, N.: A framework for expert knowledge acquisition. IJCSNS 10, 145 (2010)

7. Reinhart, G.: Digital Twin - Synchronizing Reality and Virtuality (2015)

8. Roßmann, J., Schluse, M.: Experimentierbare Digitale Zwillinge im Lebenszyklus technischer Systeme. In: Handbuch Industrie 4.0: Recht, Technik, Gesellschaft, pp. 837-859. Springer, Heidelberg (2020). https://doi.org/10.1007/978-3-662-58474-3_43

9. Schluse, M., Rossmann, J.: From simulation to experimentable digital twins. IEEE Int. Symp. Syst. Eng. 1-6 (2016)

10. Schnitger, M., Windelband, L.: Shortage of skilled workers in the manufacturing sector in Germany: results from the sector analysis (2008)

11. Tao, F., Zhang, H., Liu, A., Nee, A.Y.C.: Digital twin in industry: state-of-the-art. IEEE Trans. Ind. Informat. 15, 2405-2415 (2019). https://doi.org/10.1109/TII.2018.2873186 
Open Access This chapter is licensed under the terms of the Creative Commons Attribution 4.0 International License (http://creativecommons.org/licenses/by/4.0/), which permits use, sharing, adaptation, distribution and reproduction in any medium or format, as long as you give appropriate credit to the original author(s) and the source, provide a link to the Creative Commons license and indicate if changes were made.

The images or other third party material in this chapter are included in the chapter's Creative Commons license, unless indicated otherwise in a credit line to the material. If material is not included in the chapter's Creative Commons license and your intended use is not permitted by statutory regulation or exceeds the permitted use, you will need to obtain permission directly from the copyright holder. 